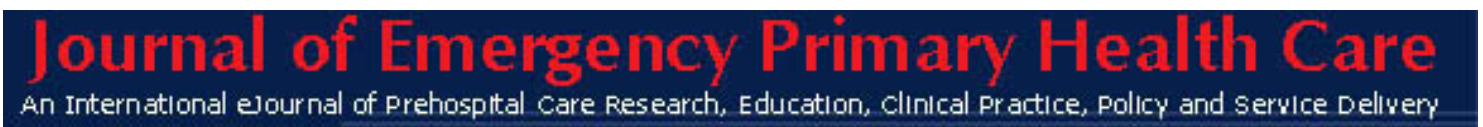

ISSN 1447-4999

\title{
PROFILE
}

Article No. 190200

\section{Vienna EMS wins International EMS Competition}

\author{
Christoph Redelsteiner, EMT-Paramedic \\ Chief of EMS \\ Vienna Red Cross \\ Nottendorfer Gasse 21
}

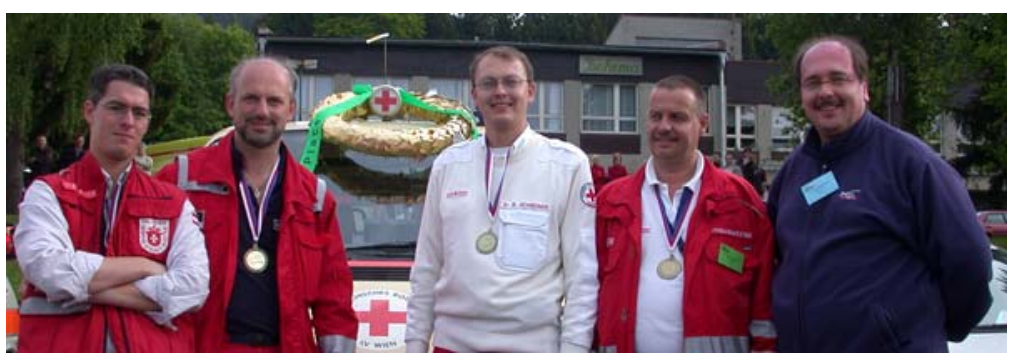

An EMS team from Vienna won the prestigious Rallye Rejviz 2006 International EMS Competition held in the Jesenik Mountains in the Czech Republic. The Rallye Rejviz (RR) was created in 1997 as part of an EMS Conference celebrating the 20th anniversary of the EMS in Jesenik - the main idea being to encourage and promote international co-operation and communication between EMS personnel at a professional level.

The event runs over one and a half days and is based on the concept of testing emergency medical skills, driving, and management skills in a friendly, yet competitive and very realistic setting.

As the term "rally" implies, competing teams have to drive from point A to point B to point C, obeying all traffic laws and without exceeding speed limits. Local police enforce the laws and any violations lead to a reduction of points. At each station, realistic scenarios are staged using actors. Some stations may occur at night and each team is required to apply theoretical and practical skills using a variety of different techniques, equipment and systems.

This year's scenarios included:

- 6 injured patients at a construction site in an amphitheatre

- rescue of two workers injured while cutting down a tree (including a $4 \mathrm{~km}$ walk with equipment)

- shooting of the lover of a betrayed husband and $\mathrm{CO}^{2}$ poisoning of the husband and his wife

- diabetic with subsequent full cardiac arrest

- changing the wheel of own ambulance (3 minutes and 38 seconds was the best time, performed by Vienna EMS) 
The evaluation criteria is similar to competitions held in the United States. Correct skills must be utilized and knowledge of emergency medical protocols, including ACLS, correct medications, and others, must be demonstrated. Safety aspects are monitored as are social interactions with the patient and bystanders. A significant difference between the RR and competitions held in the United States, is the fact that teams (whose second language is English) are also judged on patient assessment ability in English.

A unique feature of every RR is the inclusion of a disaster scenario so that groups or teams from different countries have to work together. Information from this exercise has proved to be invaluable in the planning of international disaster response.

Depending on their home nation, the various teams involved in the 2006 RR consisted of:

- ALS Paramedics

- Driver, Nurse, Paramedic

- EMT, Physician, Nurse

- EMT, Paramedic, Physician

- EMT, Nurse, Emergency Physician

Teams came from the United States, Spain, Canada, Japan, Ukraine, Netherlands, Cypruss, Greece, Slovenia, Slovakia, the Czech Republic, Poland, Hungary, and Austria.

In 2004, three EMS professionals from the Vienna (Austria) Red Cross won the 2004 international ambulance segment. This year a Vienna EMS interagency of four people (pictured above) also won. The winning team from left to right, consisted of Albert Staettermayer, EMTP (Maltese Cross); Gerhard Schuster, EMT-P (Vienna Municipal EMS, and recent medical school graduate); Berndt Schreiner, EMT-P, MD (Vienna Red Cross); Walter Schwartz, EMT-I (Vienna Red Cross). The team was managed by Vienna Red Cross Chief, Christoph Redelsteiner (far right), a US trained paramedic with licences in Austria, Germany and the US.

For further information regarding the Rallye Rejviz, please visit the official website: http://www.rallye-rejviz.com/index.php

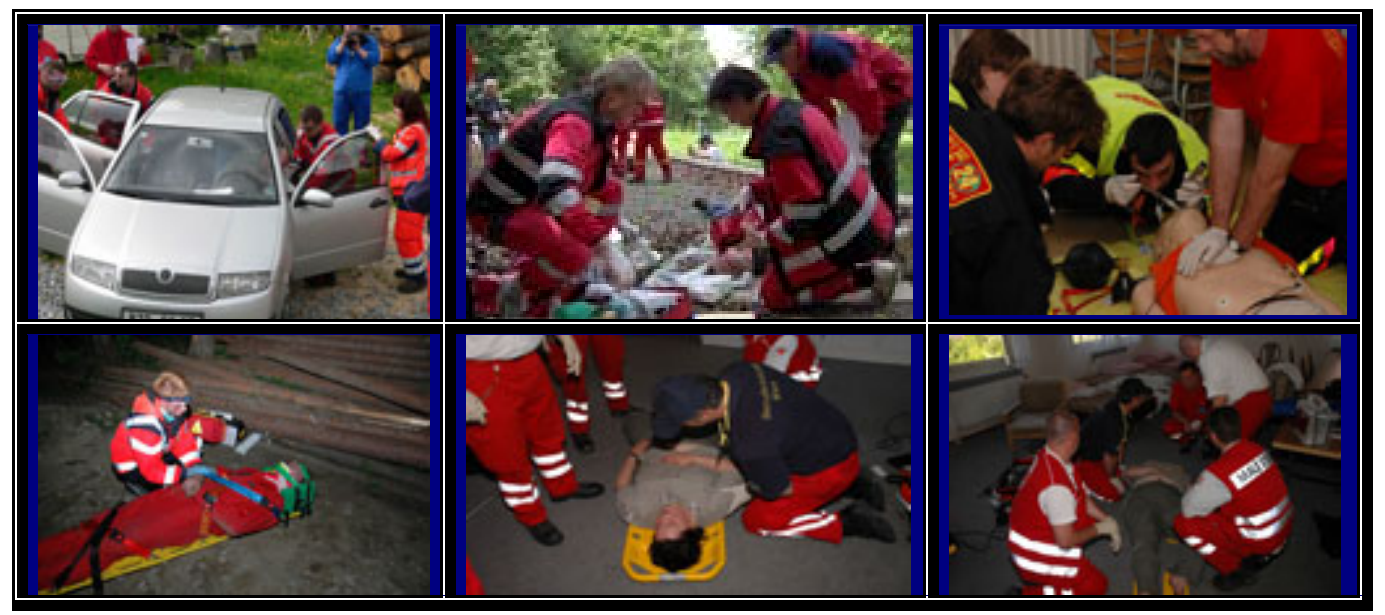

